\title{
Transport Properties of the Au-Al-Yb Quasicrystal and Approximant under Hydrostatic Pressure
}

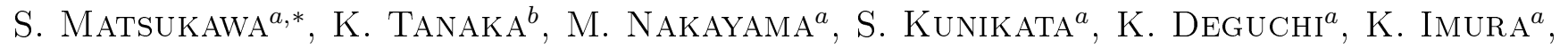 \\ T. ISHIMASA ${ }^{b}$ AND N.K. SATO ${ }^{a}$ \\ ${ }^{a}$ Department of Physics, Graduate School of Science, Nagoya University, Nagoya 464-8602, Japan \\ ${ }^{b}$ Division of Applied Physics, Graduate School of Engineering, Hokkaido University, Sapporo 060-8626, Japan
}

\begin{abstract}
The mixed-valence Au-Al-Yb quasicrystal exhibits unusual quantum criticality. Its crystalline approximant behaves like a conventional heavy fermion metal. Due to these novel features, they have attracted much attention recently. To examine the sample dependence of this new type of materials, we have prepared several samples and measured the electrical resistivity both at ambient and high pressures as well as the magnetic susceptibility at ambient pressure. The residual resistivity ratio of the quasicrystals is almost sample independent while the power law exponent $n$ of the electrical resistivity varies from sample to sample. The effective magnetic moment $p_{\text {eff }}$ of the quasicrystals is also sample dependent, ranging from about 3.4 to $3.9 \mu_{\mathrm{B}} / \mathrm{Yb}$, and these values are all smaller than the free $\mathrm{Yb}^{3+}$ ion value, confirming the mixed-valence nature. Although the magnitude of $n$ and $p_{\text {eff }}$ is sample-dependent, the principal feature of the mixed-valence is confirmed in all the samples studied thus far. A combination of these results indicates correlation between $n$ and $p_{\text {eff }}$, suggesting that the $4 f$ electrons may contribute to a scattering mechanism of the conduction electrons. External pressure increases the magnitude of the electrical resistivity and decreases the index $n$ of both the quasicrystal and the approximant. Up to the highest pressure measured in the present study no experimental evidence for a phase transition was found.
\end{abstract}

DOI: $10.12693 /$ APhysPolA.126.527

PACS: 71.27.+a, 72.15.Eb, 75.50.Kj

\section{Introduction}

Heavy fermion intermetallic compounds have attracted much interest because of their unusual behavior. The low-temperature properties of these materials differ dramatically from those of ordinary Fermi systems. For instance, the strong correlation between the $4 f$ or $5 f$ electrons contributed by the constituent elements (i.e. rare earth or actinide atoms, respectively) of the materials leads to a renormalization of the effective mass of quasiparticles; the effective mass of the heavy fermions may exceed the ordinary band mass by 2 to 3 orders of magnitude. The ground state of the heavy fermions varies with materials; some remain to be a paramagnetic Fermi liquid down to very low temperatures while some undergo an antiferromagnetic transition at a Néel temperature $T_{\mathrm{N}}$. The phase transition temperature $T_{\mathrm{N}}$ may decrease with increasing pressure $P$ and vanish to zero at a critical pressure $P_{\mathrm{c}}$. When $T$ is kept at zero temperature and $P$ is increased, the antiferromagnetic to paramagnetic transition state will occur at $P=P_{\mathrm{c}}$. If this order-disorder transition is continuous, then the critical point $(T=0$, $\left.P=P_{\mathrm{c}}\right)$ in the phase diagram is called a quantum critical point. At $T=0$ where the quantum phase transition takes place, there are no thermal fluctuations and thus the phase transition is driven by quantum fluctuations. As $T \rightarrow 0$ at fixed $P=P_{\mathrm{c}}$, such metals exhibit non-

\footnotetext{
* corresponding author; e-mail: matsukawa.shuya@h.mbox.nagoya-u.ac.jp
}

Fermi liquid behavior with unusual power laws in the temperature dependence of the physical properties; for example, the power law exponent $n$ of the electrical resistivity $\left(\rho \propto T^{n}\right)$ deviates from the Fermi liquid value of $n=2$. This non-Fermi liquid behavior is caused by the quantum fluctuations.

Until very recently, quantum criticality has been considered to be specific to crystalline materials. However, some of the present authors found a quasicrystal (QC) to exhibit novel quantum critical behavior as observed in some Yb-based heavy fermion materials [1]. This new type of quasicrystal, $\mathrm{Au}_{51} \mathrm{Al}_{34} \mathrm{Yb}_{15}$, was discovered in the course of research on a new series of Tsai-type quasicrystals [2]. By contrast, the approximant crystal (AC) shows heavy fermion feature similar to the conventional heavy fermions; note that $C_{\mathrm{M}} / T$ exceeds $1 \mathrm{~J} / \mathrm{K}^{2}$ mol-Yb at low temperatures (where $C_{\mathrm{M}}$ is the magnetic contribution to the specific heat) [1]. The absence of the divergence in the $\mathrm{Au}-\mathrm{Al}-\mathrm{Yb} \mathrm{AC}$ implies that the quantum criticality of the $\mathrm{Au}-\mathrm{Al}-\mathrm{Yb} \mathrm{QC}$ may be related to a critical state peculiar to the $\mathrm{QC}$. Interestingly, the valence of the $\mathrm{Yb}$ ion in the $\mathrm{QC}$ and $\mathrm{AC}$ is intermediate between $\mathrm{Yb}^{2+}$ and $\mathrm{Yb}^{3+}[1,3]$. The quantum critical behavior of the $\mathrm{Au}-\mathrm{Al}-\mathrm{Yb} \mathrm{QC}$ is robust against the application of hydrostatic pressure in remarkable contrast to the conventional heavy fermions.

These novel QCs and ACs have not yet been sufficiently studied. In this paper we report sample dependence of the transport and magnetic properties and attempt to find the relationship between transport and magnetism. 


\section{Experimental details}

The purity of starting materials used in the present study was as follows; Au: 99.99, Al: 99.999, and $\mathrm{Yb}$ : 99.9\% purity. To prepare the QCs these starting materials were arc-melted on a water-cooled copper hearth under an argon atmosphere. The ACs were prepared by two methods: arc-melting of the starting materials with subsequent annealing of the obtained alloy ingot in an evacuated quartz ampoule at $700^{\circ} \mathrm{C}$ for 24 hours, and the melting of the starting materials in an evacuated quartz ampoule as in annealing. Note that the alloy composition is a starting, nominal composition. Detailed description of the preparation and characterization of these and related systems is given elsewhere $[4,5]$.

Hydrostatic pressure was applied using a $\mathrm{NiCrAl}-\mathrm{BeCu}$ piston cylinder cell with Daphne oil 7373 as a pressure transmitting medium [6]. The pressure at low temperature was determined by the superconducting transition of indium that was put into the pressure cell together with a sample [7]. The electrical resistivity measurements were done using a conventional dc method in a typical temperature range between 2 and $300 \mathrm{~K}$. The dc magnetization was measured using a commercial magnetometer in terms of SQUID in a temperature range between 1.8 and $300 \mathrm{~K}$ and magnetic fields up to $7 \mathrm{~T}$.

\section{Results and discussion}

Figure 1a shows the temperature dependence of the electrical resistivity of the $\mathrm{Au}-\mathrm{Al}-\mathrm{Yb}$ quasicrystals. We observe a marked sample dependence in the magnitude of the electrical resistivity; the largest and smallest values of the resistivity differ by a factor of 5 . However, the relative temperature dependence of the resistivity seems not to be strongly dependent on the sample. To see this more clearly, we plot in Fig. $1 \mathrm{~b}$ the resistivity ratio $\rho(T) / \rho(300 \mathrm{~K})$ at low temperatures below $25 \mathrm{~K}$, where $\rho(300 \mathrm{~K})$ denotes the electrical resistivity at $T=300 \mathrm{~K}$. We observe that the residual resistivity ratio $\rho(T) / \rho(300 \mathrm{~K})$ is about $0.79 \pm 0.01$ for the samples presented here. When fitting the electrical resistivity in the lowest temperature region measured to the form of $\rho=\rho_{0}+A T^{n}$ (where $\rho_{0}$ denotes a residual resistivity), we observe the power law index $n$ to vary from sample to sample, ranging from about 1 to 0.8 (see below for details). In our previous paper, we reported that $n \approx 1$ for the $\mathrm{Au}-\mathrm{Al}-\mathrm{Yb} \mathrm{QC}$. We now find that the power law exponent $n$ slightly depends on sample. Indeed, we need to measure the resistivity at a lower temperature; this is in progress.

What is a key factor that determines the value of $n$ ? According to our preliminary results of X-ray powder diffraction patterns, we failed to find any correlation between $n$ and the (six-dimensional) lattice parameter, ranging between 0.743 and $0.745 \mathrm{~nm}$. Then, to elucidate the relationship between the transport and magnetic properties, we measured the magnetic susceptibility of the $\mathrm{Au}-\mathrm{Al}-\mathrm{Yb} \mathrm{QCs}$. The results are plotted in Fig. 2 in the form of $\chi^{-1}(T)$ vs. T. The overall feature of the inverse magnetic susceptibility (i.e., the straight
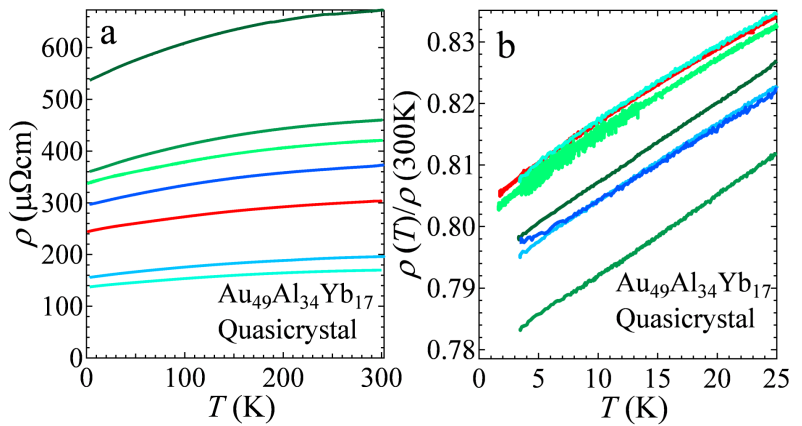

Fig. 1. (a) Temperature dependence of the electrical resistivity of the $\mathrm{Au}-\mathrm{Al}-\mathrm{Yb}$ quasicrystals. The magnitude of the resistivity shows a remarkable sample dependence. (b) Resistivity ratio $\rho(T) / \rho(300 \mathrm{~K})$ as a function of temperature.

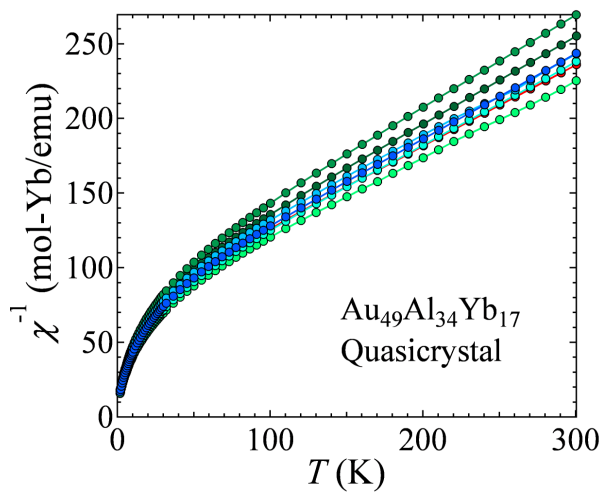

Fig. 2. Temperature dependence of the inverse magnetic susceptibility of the $\mathrm{Au}-\mathrm{Al}-\mathrm{Yb}$ quasicrystals measured at $H=5 \mathrm{kOe}$. The overall feature is the same for all the samples presented here, but the effective magnetic moment is slightly dependent on sample.

line feature at high temperatures and the convex curvature below about $50 \mathrm{~K}$ ), that is presumably ascribed to the crystal field effect [5], is commonly observed for all the samples studied here. Note that these features were also observed in the AC (see supplementary information given in Ref. [1]), suggesting that the high-temperature magnetism is not related to the absence or presence of periodicity. The linear slope of the $\chi^{-1}(T)$ curves above about $150 \mathrm{~K}$ yields an effective magnetic moment $p_{\text {eff }}$, that ranges from $\approx 3.4$ to $\approx 3.9 \mu_{\mathrm{B}} / \mathrm{Yb}$. Note that $p_{\text {eff }}$ given in Ref. [1] is about $3.9 \mu_{\mathrm{B}} / \mathrm{Yb}$. These values are all smaller than the free ion value of $\mathrm{Yb}^{3+}, 4.54 \mu_{\mathrm{B}} / \mathrm{Yb}$, confirming the mixed valence nature of the QC.

Figure 3a shows the relationship between the effective magnetic moment $p_{\text {eff }}$ and the power law exponent $n$ of the electrical resistivity, both of which are obtained above. Within the experimental accuracy, it appears that there is a correlation between $p_{\text {eff }}$ and $n$; as $p_{\text {eff }}$ increases (meaning that the valence of the $\mathrm{Yb}$ ion increases toward trivalent), $n$ decreases monotonically. This suggests the 

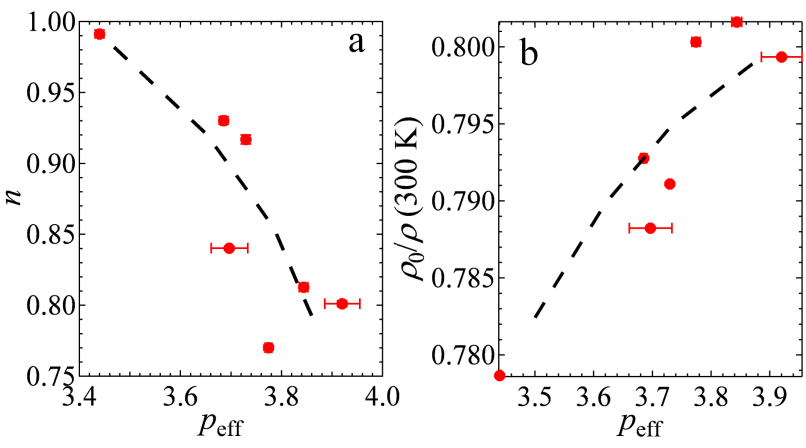

Fig. 3. (a) Relationship between the power law exponent $n$ of the electrical resistivity and the effective magnetic moment $p_{\text {eff }}$ for the $\mathrm{Au}-\mathrm{Al}-\mathrm{Yb}$ quasicrystals. (b) Relationship between the residual resistivity ratio $\rho_{0} / \rho(300 \mathrm{~K})$ and the effective magnetic moment $p_{\text {eff }}$ for the $\mathrm{Au}-\mathrm{Al}-\mathrm{Yb}$ quasicrystals. The broken lines in (a) and (b) are guides to the eye.

presence of correlation between the magnetism and the electrical resistivity. We also plot the residual resistivity ratio $\rho_{0} / \rho(300 \mathrm{~K})$ as a function of $p_{\text {eff. Again, we find a }}$ correlation between $\rho_{0} / \rho(300 \mathrm{~K})$ and $p_{\text {eff }} ; \rho_{0} / \rho(300 \mathrm{~K})$ increases with $p_{\text {eff }}$. These results indicate that the magnetic moments residing on the $\mathrm{Yb}$ ions may contribute to the scattering of the conduction electrons. It would be interesting to further study the relation between the magnetism and the transport.

Finally let us study the effect of hydrostatic pressure on the electrical resistivity. Figures $4 \mathrm{a}$ and b show the temperature dependence of the electrical resistivity of the $\mathrm{Au}-\mathrm{Al}-\mathrm{Yb} \mathrm{QC}$ and $\mathrm{AC}$, respectively, under hydrostatic pressure. The highest pressure is 12.31 and $24.26 \mathrm{kbar}$ for the $\mathrm{QC}$ and $\mathrm{AC}$, respectively. For the QC, we observe a gradual increase in the magnitude of the resistivity. The power law of the electrical resistivity of this sample is not changed so much; as shown in Fig. $5, n \approx 0.81$ at $P=$ $0.76 \mathrm{kbar}$ (the lowest pressure for this sample), and it slightly decreases to about 0.7 with increasing pressure $P$ up to $12.31 \mathrm{kbar}$. This smallness of the change in $n$ may be related to the robustness of the quantum criticality observed below $1 \mathrm{~K}$ [1]. A similar gradual increase in the magnitude of the resistivity with increasing $P$, is also observed for the AC (see Fig. 4b). The exponent $n$ of the power law of the resistivity at ambient pressure is about 1 (see Fig. 5). As the pressure increases, $n$ initially decreases to about 0.7 and then remains constant up to the highest pressure. In our previous paper, we reported that $n \approx 2$ at ambient pressure for the $\mathrm{AC}$, that led us to conclude that the $\mathrm{AC}$ is a Fermi liquid. The origin of this discrepancy is unknown at present and should be resolved in the future.

Up to the present, we find no evidence for any drastic change (such as a phase transition) in the electrical resistivity. However, at high pressures, there seems to emerge a bend-like "anomaly" that may be a signature of the transition, as marked by the arrow at $P=24.26 \mathrm{kbar}$.

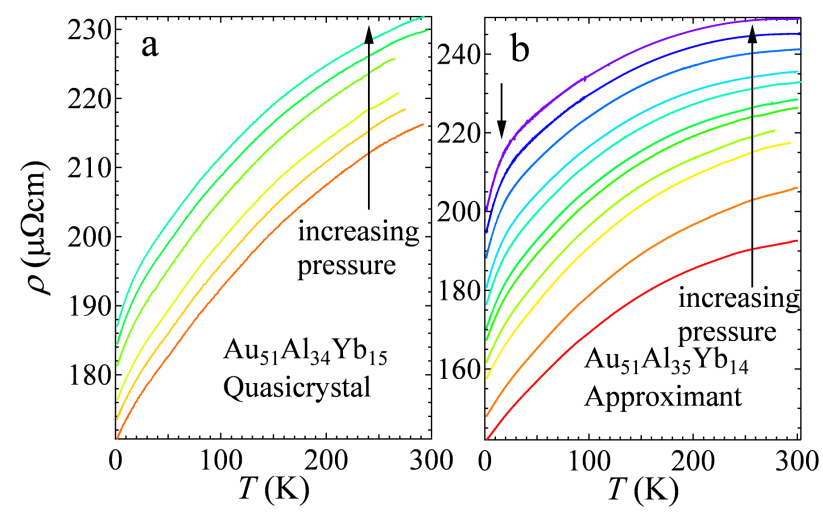

Fig. 4. (a) Temperature dependence of the electrical resistivity of the $\mathrm{Au}-\mathrm{Al}-\mathrm{Yb}$ quasicrystal. Pressure: $0.76,1.95,3.89,6.59,9.85$ and 12.31 kbar. (b) Temperature dependence of the electrical resistivity of the $\mathrm{Au}-\mathrm{Al}-\mathrm{Yb}$ approximant. Pressure: 0, 2.55, 4.15, 6.13, $8.93,9.55,12.04,15.06,17.98,21.53$ and 24.26 kbar. The arrow denotes a bend-like "anomaly".

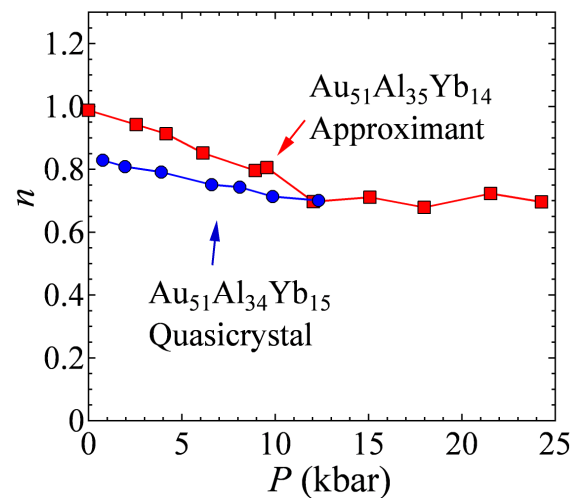

Fig. 5. Pressure dependence of the power law exponent $n$ of the electrical resistivity of the $\mathrm{Au}-\mathrm{Al}-\mathrm{Yb} \mathrm{QC}$ and AC.

To confirm the origin of this "anomaly", we need higher pressure experiments. This will be a challenging work in the future.

\section{Summary}

The mixed-valence $\mathrm{Au}-\mathrm{Al}-\mathrm{Yb}$ quasicrystal exhibits unusual quantum criticality. Its crystalline approximant behaves like a conventional heavy fermion metal with a highly enhanced effective mass [1]. Due to these novel features, the $\mathrm{Au}-\mathrm{Al}-\mathrm{Yb} \mathrm{QC}$ and $\mathrm{AC}$ have attracted much attention. To examine the sample dependence of the transport and magnetic properties of this new type of materials, we prepared several samples and measured the electrical resistivity at ambient and high pressures and the magnetic susceptibility at ambient pressure. We observed that the residual resistivity ratio $\rho_{0} / \rho(300 \mathrm{~K})$ is only weakly sample dependent while the power law exponent $n$ of the electrical resistivity varies from sample to sample, ranging from 1 to about 0.8 . The effective magnetic moment $p_{\text {eff }}$ that was deduced from 
the high-temperature linear-portion of the $\chi^{-1}(T)$ curve, was also sample dependent, ranging from about 3.4 to $3.9 \mu_{\mathrm{B}} / \mathrm{Yb}$. Although the magnitude of $n$ and $p_{\text {eff }}$ is sample-dependent, the principal feature of the mixedvalence is confirmed in all the samples studied thus far. Note that these values are all smaller than the free $\mathrm{Yb}^{3+}$ ion value, confirming the mixed-valence nature. This suggests that the difference in geometric parameters of the materials may not affect so crucially the electronic structure but the mixed valence nature is preserved. We also observed a correlation between $n$ and $p_{\text {eff }}$. This suggests that the $4 f$ electrons may contribute to the scattering mechanism of the conduction electrons. Finally we observed that the external pressure increases the magnitude of the electrical resistivity and decreases the exponent $n$ of both the quasicrystal and the approximant crystal. Up to the highest pressure measured (12 and $24 \mathrm{kbar}$ for the quasicrystal and approximant crystal, respectively), no experimental evidence for a phase transition was found.

\section{References}

[1] K. Deguchi, S. Matsukawa, N.K. Sato, T. Hattori, K. Ishida, H. Takakura, T. Ishimasa, Nature Mater. 11, 1013 (2012).

[2] T. Ishimasa, Y. Tanaka, S. Kashimoto, Philos. Mag. 91, 4218 (2011).

[3] T. Watanuki, S. Kashimoto, D. Kawana, T. Yamazaki, A. Machida, Y. Tanaka, T.J. Sato, Phys. Rev. B 86, 094201 (2012).

[4] K. Tanaka, Y. Tanaka, T. Ishimasa, M. Nakayama, S. Matsukawa, K. Deguchi, N.K. Sato, Acta Phys. Pol. A 126, 603 (2014).

[5] S. Matsukawa, K. Tanaka, M. Nakayama, K. Deguchi, K. Imura, H. Takakura, S. Kashimoto, T. Ishimasa, N.K. Sato, J. Phys. Soc. Jpn 83, 034705 (2014).

[6] K. Imura, K. Matsubayashi, H.S. Suzuki, K. Kabeya, K. Deguchi, N.K. Sato, J. Phys. Soc. Jpn. 78, 104602 (2009).

[7] L.D. Jennings, C.A. Swenson, Phys. Rev. 112, 31 (1958). 